\title{
Power-Steering Control Architecture for Automatic Driving
}

\author{
Jóse E. Naranjo, Carlos González, Member, IEEE, Ricardo García, Teresa de Pedro, and Rodolfo E. Haber
}

\begin{abstract}
The unmanned control of the steering wheel is, at present, one of the most important challenges facing researchers in autonomous vehicles within the field of intelligent transportation systems (ITSs). In this paper, we present a two-layer control architecture for automatically moving the steering wheel of a mass-produced vehicle. The first layer is designed to calculate the target position of the steering wheel at any time and is based on fuzzy logic. The second is a classic control layer that moves the steering bar by means of an actuator to achieve the position targeted by the first layer. Real-time kinematic differential global positioning system (RTK-DGPS) equipment is the main sensor input for positioning. It is accurate to about $1 \mathrm{~cm}$ and can finely locate the vehicle trajectory. The developed systems are installed on a Citroën Berlingo van, which is used as a testbed vehicle. Once this control architecture has been implemented, installed, and tuned, the resulting steering maneuvering is very similar to human driving, and the trajectory errors from the reference route are reduced to a minimum. The experimental results show that the combination of GPS and artificial-intelligence-based techniques behaves outstandingly. We can also draw other important conclusions regarding the design of a control system derived from human driving experience, providing an alternative mathematical formalism for computation, human reasoning, and integration of qualitative and quantitative information.
\end{abstract}

Index Terms-Architecture, fuzzy control, global positioning, intelligent control, road vehicle control.

\section{INTRODUCTION}

$\mathbf{N}$ OWADAYS, the development of intelligent transportation systems (ITSs) provides an opportunity to apply advanced technology to systems and methods of transport for efficient, comfortable, and safer highways, railways, inland waterways, airports, ports, and linkages between all these means of transport [1]. Our work focuses on the area of road transport, and more specifically, on the field of intelligent vehicles, which includes the issue of autonomous vehicles. These vehicles are equipped with the instrumentation and the intelligence necessary to perform the required task, since an autonomous car must control some or all of its functions without external intervention. In this case, our aim is the automatic control of

Manuscript received January 13, 2005; revised May 17, 2005. This work was supported in part by the Spanish Ministry of Education under Grant ISAAC CICYT DPI2002-04064-C05-02, the Spanish Ministry of Public Works under Grant COPOS BOE 280 November 22, 2002. Res. 22778, and the Citroën España S.A. under Contract "Adquirir nuevos conocimientos sobre la introducción de las tecnologías de la información en el mundo del automóvil y para difundirlos en los ámbitos científicos, empresariales y comerciales (Autopia)." The Associate Editor for this paper was R. Goudy.

The authors are with the Industrial Computer Science Department, Instituto de Automática Industrial (CSIC), La Poveda-Arganda del Rey, 28500 Madrid, Spain (e-mail: jnaranjo@iai.csic.es; gonzalez@iai.csic.es; ricardo@iai.csic.es; tere@iai.csic.es; rhaber@iai.csic.es).

Digital Object Identifier 10.1109/TITS.2005.858622 the steering wheel (also known as lateral control) of a massproduced vehicle, although another area of our research is longitudinal control, that is, the control of the vehicle's speed and its adaptation to road features, using the throttle and the brake pedal as needed [2], [3].

The actual implementation of full automatic-steering control is one of the disciplines in the intelligent-vehicles field that is receiving less attention now than it did some years ago. The reason for this is that perhaps it has a long way to go before it comes on the market [4], and vehicle manufacturers focus on more mature systems, especially for speed automation, some of which are already available on the market.

There is, however, a short-term focus to steering control, albeit not for lateral guidance, as part of a driving assistance system, similar to an electronic stability program (ESP). Ackermann in [5] compares the performance of the two systems, finding that the steering-control method is more reliable, because it has a quicker effect on vehicle stability.

Automatic parking systems [6] are other steering-control applications that are already on the market. These systems exploit the preinstalled vehicle electric power-steering systems [7] to automatically manage the steering wheel for parking purposes.

There can be no doubt, however, that full steering management for vehicle lateral-control applications is the realm of pure research. Some autonomous-vehicle steering control has been developed and implemented, and experiments have been successful.

There are two ways to design steering controllers: imitating human drivers and using dynamic models of car and control methods based on linear control theory. The first approach does not need detailed knowledge of car dynamics, much in the way the driver of a car does not. In this case, the algorithm mimicking human driving behavior is the key to control. The control-system approach requires detailed knowledge of the dynamics of the car and has to use different algorithms to perform the different maneuvers. Ride quality, which should be considered in steering control, is more or less a built-in feature of the first approach. It is, however, very difficult to introduce in the second one.

According to literature, the first automated steering wheel was built in 1977 in Japan, as part of the Comprehensive Automobile Traffic Control System (CACS) project, under the direction of Professor Tsugawa [8]. This was a primitive control system based on embedded hardware that could manage the steering-wheel movement for short routes (of about $50 \mathrm{~m}$ ). It used a motor attached to the steering wheel and was based on artificial vision and proportional control. 
More modern developments are able to manage this actuator on normal roads, adapting the vehicle cruise for drivinglane tracking, where automatic driving mimics human driving. Prof. Tsugawa and his group keep researching on autonomous vehicles, having developed controllers for lateral and longitudinal control, which are mainly based on classical geometric control global positioning system (GPS) and intervehicle communications [9].

Similarly, within the ARGO project developed at Parma University by Prof. Broggi's team, a vehicle was instrumented with artificial-vision cameras and a PC-based computer in order to manage the steering wheel automatically on routes along public highways [10]. The guidance system is based on a classical P controller, the input signals of which are directly supplied by the lane-recognition vision system [11].

Chaib et al. describe in [12] the work done on automatic lateral control within the French Institut National De Recherche sur les Transports et Leur Securite (INRETS)/Laboratoire Central des Ponts et Chaussées (LCPC) Laboratoire sur les Interactions Véhicules-Infrastructure-Conducteurs (LIVIC), comparing $\mathrm{H} \infty$, adaptive, proportional-integral differential (PID), and fuzzy controllers, and outlining the advantages and drawbacks of using these techniques. They have all been tested on real vehicles.

Carnegie Mellon University's Navigation Laboratory (NavLab) laboratory has gained a lot of experience in developing steering controllers for the NavLab vehicle series. These vehicles are equipped with artificial-vision systems, and the steering in the first versions was controlled by the neural-network-based rapidly adapting lateral position handler (RALPH) [13].

Several lateral controllers have been developed in the Partners for Advanced Transit and Highways (PATH) program [14]-[17]. One such controller is described in [18], where Hessburg and Tomizuka present a lateral-vehicle-guidance system based on fuzzy control that has been installed in an experimental test Toyota Celica vehicle. There are actually many reasons for using fuzzy control to manage the guidance of a vehicle. The first is that the controller incorporates human knowledge and engineer judgments. The second is that the rule base can be designed to include all the nonlinearities associated with the vehicle's dynamics, like tire characteristics or mechanical variations [19].

In particular, Sugeno and Nishida [20] demonstrated that fuzzy control was capable of handling nonlinear control problems to maneuver a model car using oral instructions.

Some other real-vehicle applications that are capable of autonomous-steering management and performing humanlike tracking have been developed ([21]-[23]). The main field of interest for this research is sensor equipment and perception of the driving area.

A car is an example of typical system where driving models, sensory information, objectives, constraints, and control actions are essentially inaccurate. We have used fuzzy logic to design lateral controllers in the Autopia program. The main reason for using this approach is that a suitable driving-process model is essential for automatic steering-wheel control. Nevertheless, classical approaches frequently fail to yield appropriate models of complex (nonlinear, time-varying, ill-defined) processes, which driving a car certainly is, while fuzzy-logic-based control methods provide an alternative tool for dealing with car and subsystem complexity. The Autopia program kicked off in 1996 at the Industrial Automation Institute (IAI), a division of the Spanish Council for Scientific Research (CSIC), and is funded by several national research projects in the field of ITS and private companies. The main goal of Autopia is to develop unmanned vehicles for specific applications, and research is focused on mass-produced cars. Some systems for controlling both car speed [2], [3] and steering [24] have been developed, using GPS as the main sensor input for navigation, and wireless communication for sharing information among all the vehicles on the road. The controls have been installed in two Citroën Berlingo vans that have automated actuators (steering, throttle, and brake) and tested on a private circuit at the IAI facilities. This private circuit emulates the structure of an urban area. The reason for this is that urban autonomous driving is one of the most difficult and least researched subjects for intelligentvehicle applications.

In this paper, we present the automatic-steering control architecture, based on a combination of fuzzy logic and PID control, for managing the lateral guidance of a vehicle as human drivers would do. To the best of our knowledge, the main contribution of this work is the combined use of a PID and a fuzzy controller in a cascade-control scheme, and its application to regulate the steering wheel of a mass-produced vehicle.

This paper is organized as follows. Section II shows the Autopia steering control architecture, covering vehicle instrumentation and lateral-control organization. Section III addresses experiments with the control and, finally, some concluding remarks are given.

\section{Autopia Steering-Control-Architecture DEFINITION}

\section{A. Vehicle Instrumentation}

The Citroën Berlingo sensor equipment for automatic driving is composed of a real-time kinematic differential GPS (RTKDGPS) receiver, the antenna of which is mounted over the vehicle rear axle. This sensor uses the carrier phase of the GPS signal, complemented with the positioning correction supplied by a GPS base station located near the driving zone, to provide a to-the-centimeter positioning precision at a rate of $10 \mathrm{~Hz}$. This precision is relative to the location of the base. Therefore, yielded positions are as accurate as the base position is, albeit perfectly repeatable. Although precision can be affected by a number of factors such as the visibility of the GPS satellite constellation, the local topography, the season, and the link used to feed the GPS differential corrections, the potential of the application of RTK-DGPS for autonomous driving is demonstrated in this paper. There are several techniques for overcoming these shortcomings such as including inertial systems [25]. Navigation is based on GPS maps. We build our maps driving along a track recording the GPS positions, as it is common in many applications [26]. Thus, our maps are as good as our measures, but they are always coherent with them. However, if we use absolute coordinates taken from external 


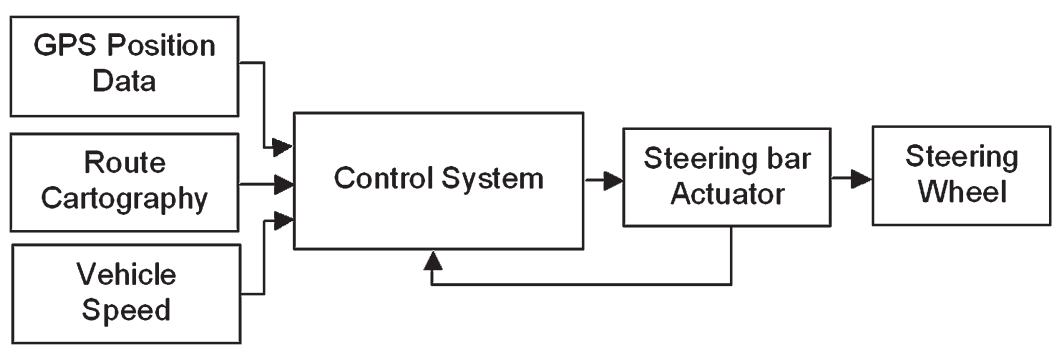

Fig. 1. Steering automation architecture.

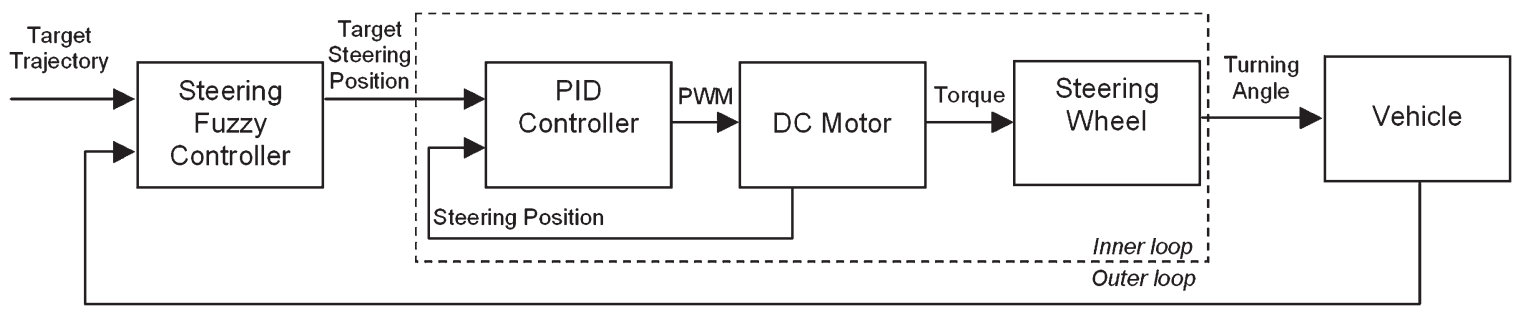

Fig. 2. Steering cascade-controller diagram.

maps, we should check it to ensure the coherence between measured values and absolute values. For trajectory control, the position data are compared with the maps that define the target route to be tracked. Each map indicates a reference trajectory over the area with GPS base-station coverage. Precision of the GPS measure decreases with the distance to the base station, and problems might arise should we deal with great distances.

Navigation computation runs on an onboard industrial computer that also houses the control system that manages the lateral or steering control. This computer is able to command a DC motor that is attached to the steering-wheel bar by means of gears. This motor can move the steering wheel as fast and as accurately as a human driver does. Finally, an analogical input card reads the speed information from the vehicle, which is also used as the input for the control system.

A diagram of the control architecture is shown in Fig. 1, illustrating the four inputs that the control system needs: GPS, route, speed, and steering-wheel position. The control system commands the steering-wheel actuator and receives from it the actual steering-wheel position.

The lateral control system, which is described below, is the core of the architecture.

\section{B. Lateral-Control Organization}

A two-layer control architecture has been designed to manage the steering of our vehicles. A high-level layer based on a fuzzy controller is in charge of acquiring the sensor information and comparing it with the reference trajectory to generate a target steering-wheel turning command. The low-level layer is composed of an LM629 hardware motor control card [27], in which a classical PID controller receives the high-layer output turning command and manages the motor attached to the steering bar to take it to the reference position. This two-layer architecture has been organized on the basis of the cascadecontrol-architecture paradigm [28].
1) Cascade-Control Paradigm: The cascade-control paradigm is particularly useful when there is a significant time delay between the action variable and the controlled variable. In this case, tighter control can be achieved using an intermediate measurement signal that responds faster to the control signal. Two loops are involved in the control scheme. An inner loop, also known as secondary loop, and an outer loop, known as primary loop.

The control diagram for our case is shown in Fig. 2, where the primary loop is in charge of determining the target steeringwheel position, refreshing it at GPS output speed, and the secondary loop tracks the turning of the motor to reach this target position in a faster loop.

2) Outer Loop: Steering-Position Fuzzy Controller: The objective of the outer loop is to acquire the related input data from the sensors and then process this information to generate a reference position to be reached by the steering wheel. The sensor input comes from the GPS receiver that locates the vehicle in the driving area at a $10-\mathrm{Hz}$ sampling rate. Each measured value is compared with a reference GPS trajectory that defines the route to be taken. Two input variables are gathered from this comparison: the lateral and the angular errors from the reference route. A fuzzy controller is the main subsystem or component of the primary loop and manages both input variables in order to generate the target steering turning command. This fuzzy controller performs the three basic tasks known as fuzzification, inference, and defuzzification. Fuzzification is the stage in which the input crisp values are transformed into fuzzy data. Inference is the procedure whereby the values of the fuzzy variables are inferred from a rule base, generating a fuzzy value for the output variable: the target steering-wheel angle. The final stage, defuzzification, transforms this output fuzzy value into crisp data that can be sent to an actuator or to the secondary loop.

a) Inputs and output: Only two input variables are needed to manage the steering wheel of a vehicle: the lateral error and the angular error. 


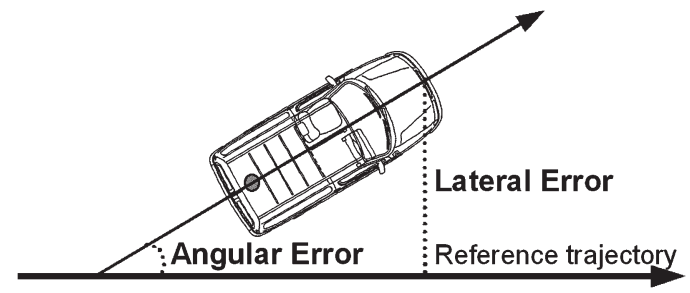

Fig. 3. Illustration of input variables.

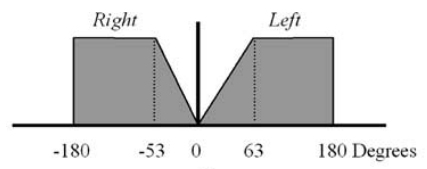

c1)

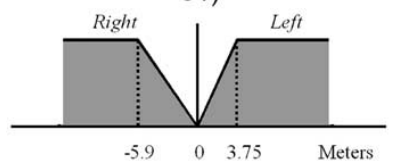

c2)

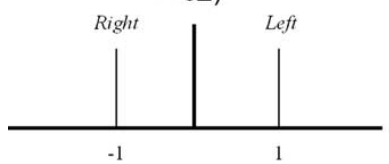

c3)

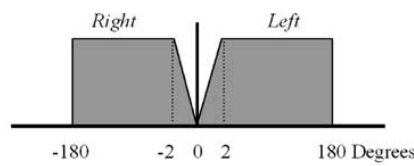

s1)

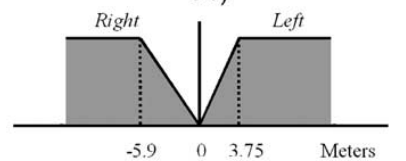

s2)

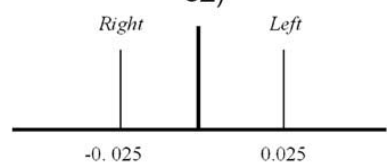

s3)
Fig. 4. Fuzzy-variable membership functions. Straight-road controller input: s1) Angular error. s2) Lateral error. Output: s3) Steering turning. Curvedroad controller input: c1) Angular error. c2) Lateral error. Output: c3) Steering turning.

The lateral error is the deviation, in meters, of the front of the car from the reference trajectory, measured perpendicularly from it. The angular error is the angular deviation, in degrees, of the vehicle from the reference trajectory and is represented by a director vector. Fig. 3 illustrates these variables.

The output of the fuzzy controller will be the target turning sent to the low-level controller as a reference. In this case, this value is absolute and indicates a steering angle.

To manage the input variables in a fuzzy context, their crisp values have to be fuzzified. We have defined two fuzzy variables for the lateral and angular errors, the membership functions of which are associated with some linguistic labels. Similarly, the fuzzy output variable must be defined in a fuzzy context. Each of the fuzzy variables has two associated linguistic labels, left and right, which will be used depending on what side the errors in the input variables fall or the direction of the output. The definition of these fuzzy variables is shown in Fig. 4.

Two operating modes are contemplated in the steering control, i.e., straight-road driving and curve driving. These modes influence the membership-function definitions. The left column of Fig. 4 shows the membership-function definitions and the associated linguistic labels corresponding to the curve-driving variables and the right column shows those for straight-line driving. All of these shapes are derived from the verbal description of driver behavior, i.e., they are clearly intuitive. However, they are refined experimentally [29]. This way, when driving along a straight road, the movements of the steering wheel should be fast and short like that of a human, that is, very reactive. The definition of the membership functions for the straight-driving steering controller accounts for this feature. Reactivity is achieved by the membership functions of the input variables having a sharper definition, and the short turnings are provided by the definition of the output variable as a singleton (s3), which limits turnings to $2.5 \%$. This means that if the maximum steering-wheel turning is $\pm 540^{\circ}$, the turning limit is $12.5^{\circ}$ for straight-road driving. Considering the Lateral_Error and Angular_Error definitions in Fig. 4, s1 and s2 represent the degree of truth for the input error values in straight-path tracking situations. The linguistic labels right and left of the fuzzy-variable Lateral_Error show that, if the separation is greater than $0.8 \mathrm{~m}$, the degree of truth is considered 1 , this degree becoming 0 as the distance shortens. In a similar way, the linguistic labels right and left of the fuzzy-variable Angular_Error show the degree of truth to be 1 if the angle is greater than $2^{\circ}$, becoming 0 as the angle tends to 0 .

In the curve mode, the steering wheel can be turned as far as it will go, and its reactivity and speed should, of course, be lower to prevent accidents. The full turning is provided by the output-variable membership functions, extended to the $\left[-540^{\circ}, 540^{\circ}\right]$ interval, and the lower reactivity and speed by the softer definition of the input-variable membership functions. Parts c1 and c2 of Fig. 4 correspond to driving on curves and their gradients are lower. They are therefore less reactive and adapt to the route smoothly. The output $\mathrm{c} 3$ is also represented as a singleton, but, in this case, the total steering-wheel turning is permitted when necessary.

An essential characteristic of this definition of the input fuzzy variables is that their membership functions are symmetrical in the straight-road context and are not in the curve mode. The reason for this is that the main goal in straight-road driving is to track the trajectory with low errors to both the left and right. Therefore, the control response is the same in either direction [29]. On the other hand, when we are driving in the right-hand lane of a road, taking a bend to the left is different from taking a turning to the right, especially when the curvature radii are low. This is the reason why we have sharpened the membership functions for the right errors and softened the functions for the left errors. It is also closely related to the fuzzy-rule definitions, which are described next.

b) Fuzzy rules: Fuzzy rules are the controller component that accommodates the human driving experience. In our case, we have defined four intuitive rules that perfectly describe the behavior of people when maneuvering a car:

R1 IF Angular_Error Left THEN Steering Right

R2 IF Angular_Error Right THEN Steering Left

R3 IF Lateral_Error Left THEN Steering Right

R4 IF Lateral_Error Right THEN Steering Left

where fuzzy variables are in bold and the linguistic labels in italics. The rules for the controller are the same in all situations; the ones that differ are the linguistic values associated with the fuzzy variables.

The aim of the rules is to mimic human behavior when there are deviations in the trajectory: when the trajectory deviates to the right, the driver must turn the steering wheel to the left to correct this, whereas the driver should move the wheel in the opposite direction when the deviation is to the left. This explains why we have defined dual rules for both input variables. 
The extent of the turning will depend on the correct tuning of the variable membership functions and the selected defuzzification method. We use the center-of-area method shown in (1), which has proved to be the most useful method for our purposes [30]:

$$
\frac{\sum w_{i}^{2} o_{i}}{\sum w_{i}}
$$

This formula is applied to each output variable for its linguistic label $i$. The $o_{i}$ elements are the crisp values of each linguistic label $i$ of the named output variable and $w_{i}$ are the weights the linguistic values $i$ are assigned, which are computed using Mamdani's rule inference method-because its results have been widely tested in control systems-where OR is represented by the maximum operator and AND performs the minimum operation.

In our case, the only output variable is Steering and its two associated linguistic labels are Left and Right (Fig. 4, c3 and s3). Thus, the particularization of (1) becomes

$$
\text { Steering_Crisp }=\frac{O_{\text {Left }} \cdot W_{\text {Left }}^{2}+O_{\text {Right }} \cdot W_{\text {Right }}^{2}}{W_{\text {Left }}+W_{\text {Right }}} .
$$

Having described the fuzzy rules, we can clearly see the reason for the asymmetry of the curve-mode input-variablemembership-function definitions. Angular_Error Left and Lateral_Error Right are part of the rules that take nonnull values upon right turnings, whereas Angular_Error Right and Lateral_Error Left take nonnull values upon left turnings. Then, both couples of membership functions have been tuned accordingly to implement different behaviors for both turnings. In this case, the shapes defined for right turnings have been defined with flatter slopes than the ones for the left turnings. When the car nears a right turn, it cannot take the turning immediately because the corner would get in the way. The flatter slope of the functions has the effect of delaying the steering-wheel turning to achieve this effect. When the turning is to the left, there is no corner, but there is left-lane invasion, which is allowed to some extent. This effect is shown clearly when the curve radius is very short, being lower the greater the radius is, because fuzzy variables do not get to reach the top values.

3) Inner Loop: PID Controller: The trajectory control described above is unable to effectively command a position on the steering wheel. The car has hydraulic steering, and a gear and a motor had to be fitted to the steering column to control it. This motor is controlled by a classical PID-amplification motor-gearbox schema. A hardware PC control card based on an LM629 chip [27] contains this PID controller and generates a motor command in sign and magnitude [pulsewidth modulation (PWM)]. An H-bridge first receives and then sends and amplifies this command to move the motor effectively. This motor is attached to a gearbox and an encoder. The gearbox transmits the right torque to the steering bar and the encoder generates the motor position to be used as feed-forward data.

The inner loop continuously tracks the motor trajectory to perform a fine control at $100 \mathrm{~Hz}$. The PID controller was tuned on the basis of Ziegler-Nichols method and further refined ex-
TABLE I

PERFormance INDEXES OF THE PID CONTROLler IN RESPONSE TO STEP Disturbances IN THE STEERING ANGLE

\begin{tabular}{lllll}
\hline \hline $\begin{array}{c}\text { Steering } \\
\text { Angle }\end{array}$ & \multicolumn{1}{c}{ IAE } & ISE & ITAE & $\mathrm{T}_{\mathrm{ss}}$ [sec.] \\
\hline 540 deg. & 22.89 & 1.77 & 2.28 & 4.5 \\
156 deg. & 11.23 & 0.87 & 1.12 & 2.3 \\
15 deg. & 4.01 & 0.31 & 0.40 & 1.2 \\
\hline \hline
\end{tabular}

perimentally. Controller parameters (i.e., proportional, integral, and derivative gains) were adjusted to yield an overdamped closed-loop response, avoiding the negative effects of the overshoot and oscillations. The proportional, integral, and derivative gains were set at 20,50, and 1000, respectively.

Various performance indexes, such as integral of absolute errors (IAE), integral of square errors (ISE), and integral of time per absolute errors (ITAE), were calculated to assess inner loop control performance, according to

$$
\begin{aligned}
\text { ITAE } & =\int_{0}^{T} t \cdot \mathrm{e}(t) \mathrm{d} t \\
\text { ISE } & =\int_{0}^{T} \mathrm{e}^{2}(t) \mathrm{d} t \\
\text { IAE } & =\int_{0}^{T}|\mathrm{e}(t)| \mathrm{d} t .
\end{aligned}
$$

The overshoot and the settling time $T_{\mathrm{SS}}$ were also calculated.

Table I summarizes the behavior of the abovementioned PID control-system performance indexes in response to different step disturbances in the steering angle. Error-performance indexes are normalized with regard to the respective setpoints for the purpose of comparison.

Fig. 5 shows the step response for the three cases analyzed. First, let us consider the error-performance indexes. PID performs very well on the basis of all criteria. Likewise, neither overshoot nor oscillations appear in the closed-loop response and, therefore, an adequate transient response is obtained for all three cases. It is noteworthy that the first case considered (i.e., $540^{\circ}$ ) is not frequent in road driving, and it is the maximum turning allowable by steering wheel of our testbed vehicle. On the other hand, the second and third cases are typical turning angles for curve and straight driving, respectively.

\section{EXPERIMENTS}

This control scheme was implemented and tested in our Citroën Berlingo testbed vans. The resulting experiments showed that first-rate humanlike trajectory tracking is feasible, and very good results were achieved.

One of these tests is shown in Fig. 6. It illustrates the map, plotted in GPS coordinates, of the private driving circuit at the IAI facilities, which represents an inner city area, with a combination of straight-road segments and $90^{\circ}$ crossroads. The dotted lines that meet at the corners of the road represent the 


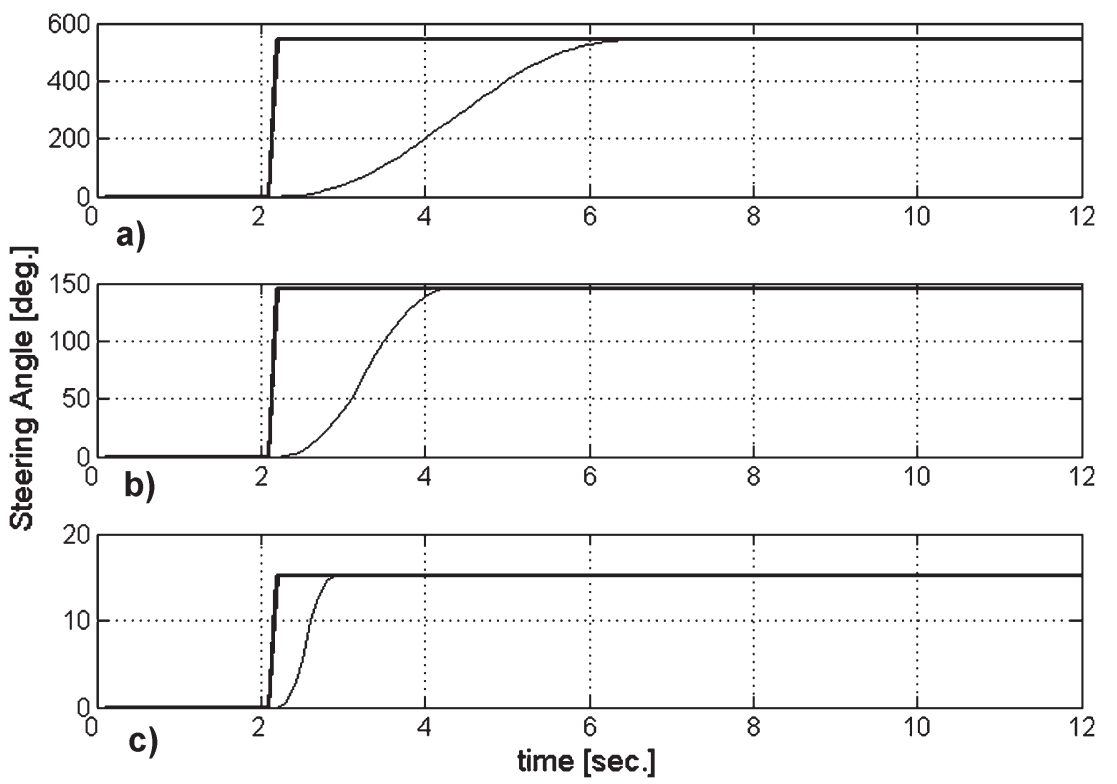

Fig. 5. Close-loop response of the PID control system in response to step disturbances in the steering angle: (a) $540^{\circ}$; (b) $146^{\circ}$; and (c) $15^{\circ}$.

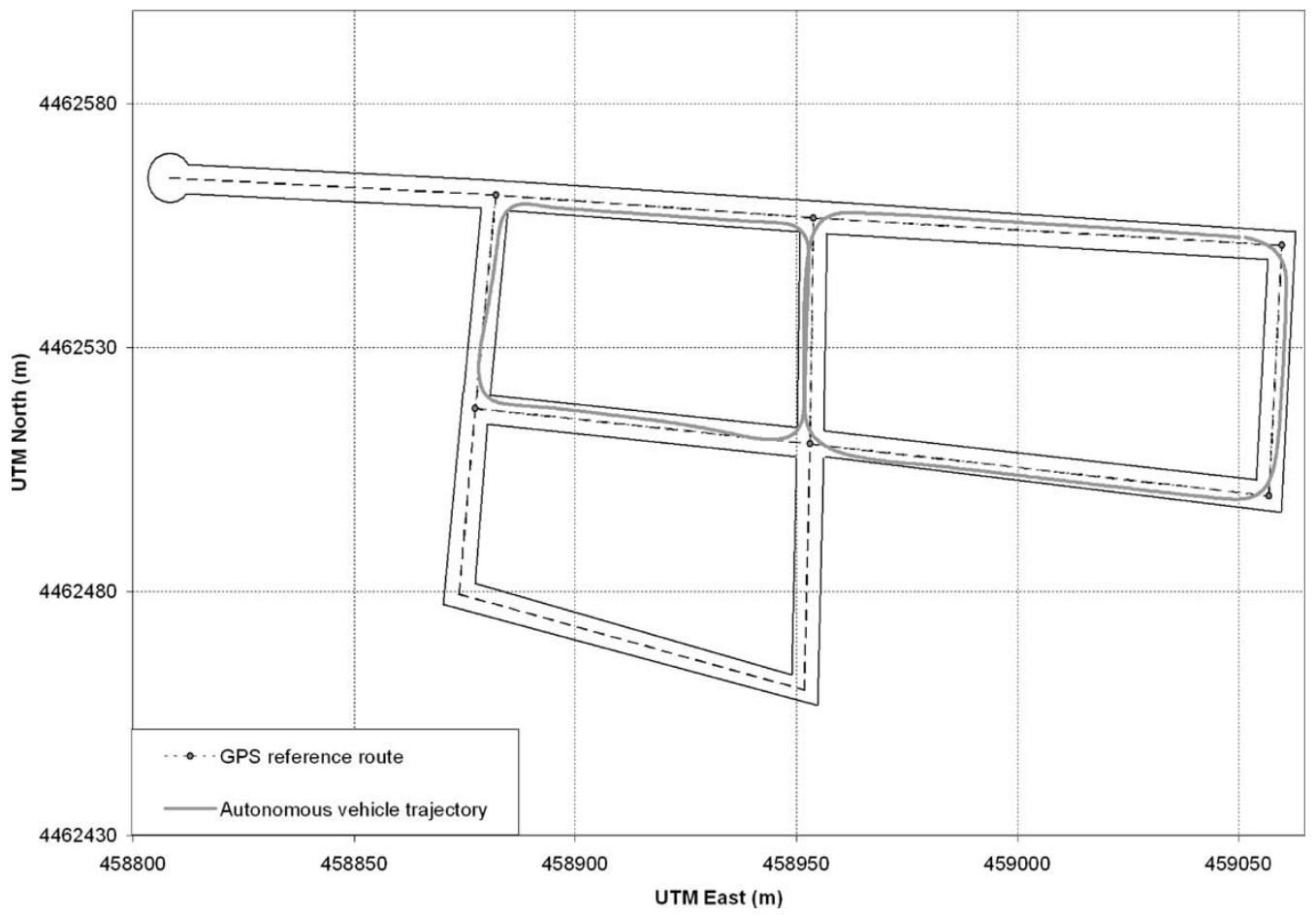

Fig. 6. Automatic-driving route experiment with $90^{\circ}$ short-radius curves.

GPS route that is used as a reference by the driving system, and the gray line symbolizes the route taken by the autonomous vehicle driven by the described controller as recorded by the on-board GPS antenna, which is located over the center of the rear axle.

In this case, the route starts at the 459050 -m East coordinate (see Fig. 6) and always keeps to the right-hand lane of the road. This path is composed of consecutive straight and bend sections (one bend to the left, four to the right and three to the left) bringing the vehicle to the vicinity of the starting location.
The map shows how the vehicle tracks the straight segments as well as the bends perfectly, always keeping to its lane and taking left and right bends differently, as necessary and defined in the input fuzzy-variables membership functions. The bend context of the fuzzy controller completes the bend tracking without going off the road and points the vehicle in the right direction along the new straight segment. On the other hand, the straight context of the controller maintains the route with average angular and lateral errors of $0.8^{\circ}$ and $0.1 \mathrm{~m}$, respectively, the maximum deviations being $3.6^{\circ}$ and $0.4 \mathrm{~m}$, which are smaller errors than humans make when driving. 

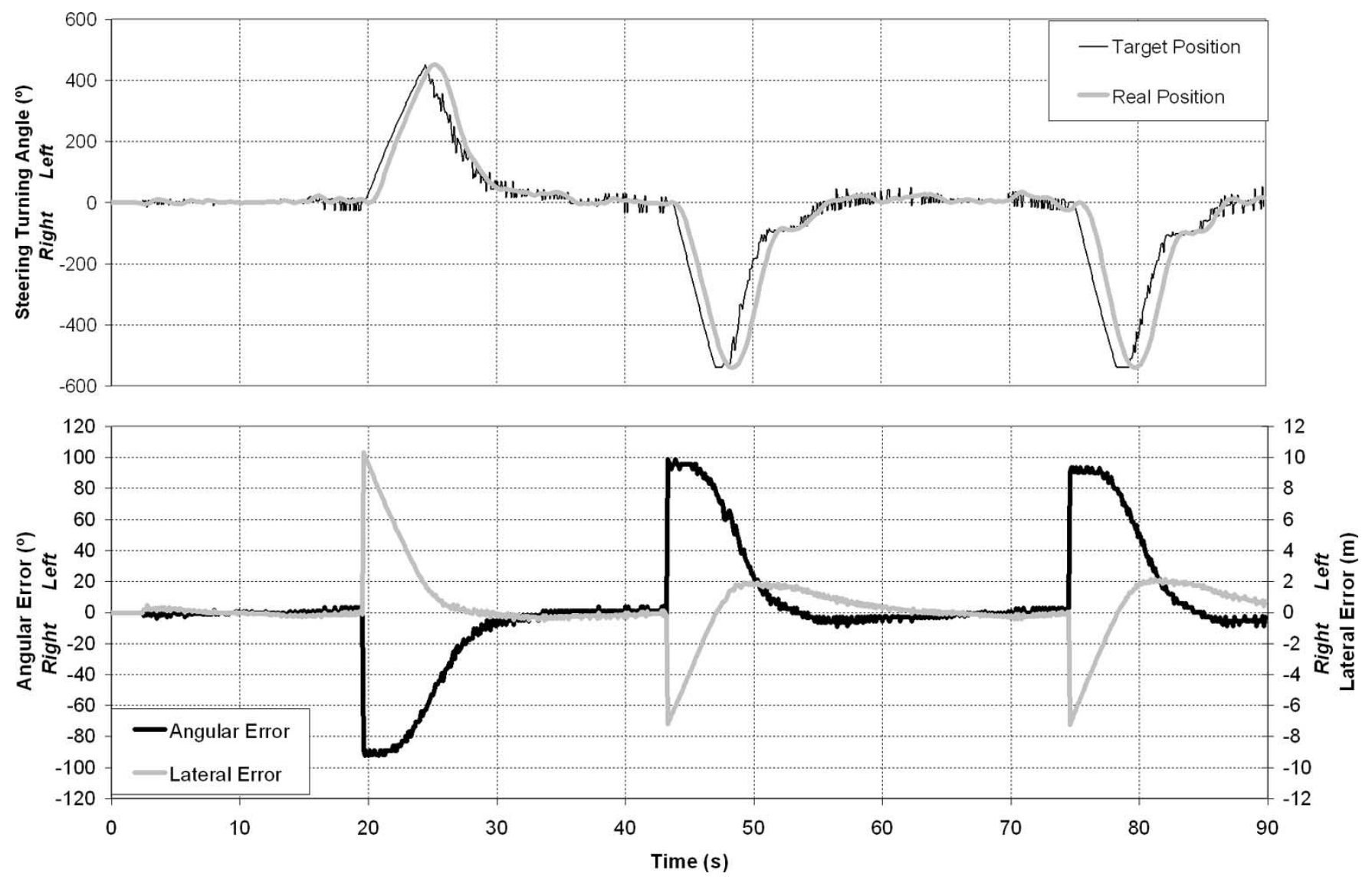

Fig. 7. Control response to the input variables during the first experimental track.

Now, we will analyze the low-level control-system behavior in this experiment. We will analyze the tracking of the first three bends, comparing the evolution of the low- and high-level controllers of the proposed cascade architecture.

Fig. 7 shows the internal values of the input and output variables for this experiment. The bottom graph represents the crisp values of the lateral and angular errors for this section of the track and the top graph contains the target steering position, generated for these fuzzy-controller inputs, and the real steering position, calculated in response to this target by the PID controller.

The track starts in a straight segment that generates low trajectory errors, corrected by the control system with short and fast steering movements.

When the car is near the first bend, the reference segment changes to the next street. Then, logically, the lateral and angular errors suddenly increase drastically. In this case, it is a left turning, and the angular error is therefore to the right and the lateral error is to the left of the new reference trajectory. In this case, rules R2 and R3 act simultaneously. At the beginning of the turning operation, both errors are very high. The lateral error is about $10 \mathrm{~m}$ (this is the reference trajectory changing distance), and the angular error is about $90^{\circ}$, because the new street is perpendicular to the one the vehicle is moving along. As is inferred from Fig. 7, the error-induced control actions cancel themselves out, and the output of the fuzzy controller does not move the steering wheel initially. As the car approaches the new street, the lateral error drops and the angular error remains the same. Consequently, the weight of $\mathrm{R} 2$ in the control action increases and starts to turn the steering wheel to the left. This action implies that both errors continue to fall: the lateral error approximates the vehicle to the new reference and the angular error points the car in the right direction. The correct tuning of the membership functions of both variables assured that the turning was correct, and the car ended up centered in the new reference lane.

The output of the steering fuzzy controller, the target turning angle, is sent to the secondary-loop PID controller, the tracking error being minimum and complying perfectly with its mission, that is, to move the steering wheel to the position commanded by the fuzzy controller fast and accurately enough to keep the car in the right trajectory. Fig. 7 shows that the inner controller behaves properly in both the straight and bend modes.

Once centered in the new street, the fuzzy context changes again to straight mode and driving continues towards the next bends in the circuit, which are, in this case, to the right, with the fuzzy controller also being able to take them correctly.

The second experiment shows control-system performance when the reference route is composed of a set of bends with different curvature radii. This experiment is illustrated in Fig. 8. In this case, the radius of the bends is between 10 and $30 \mathrm{~m}$, and there are consecutive bend segments with different curvatures.

In the experiment, the vehicle starts its automatic route in the middle of the longest street of the circuit and always circulates in the near-3-m-wide right lane of the road. Tests corroborate that the vehicle keeps to the reference route correctly, without 


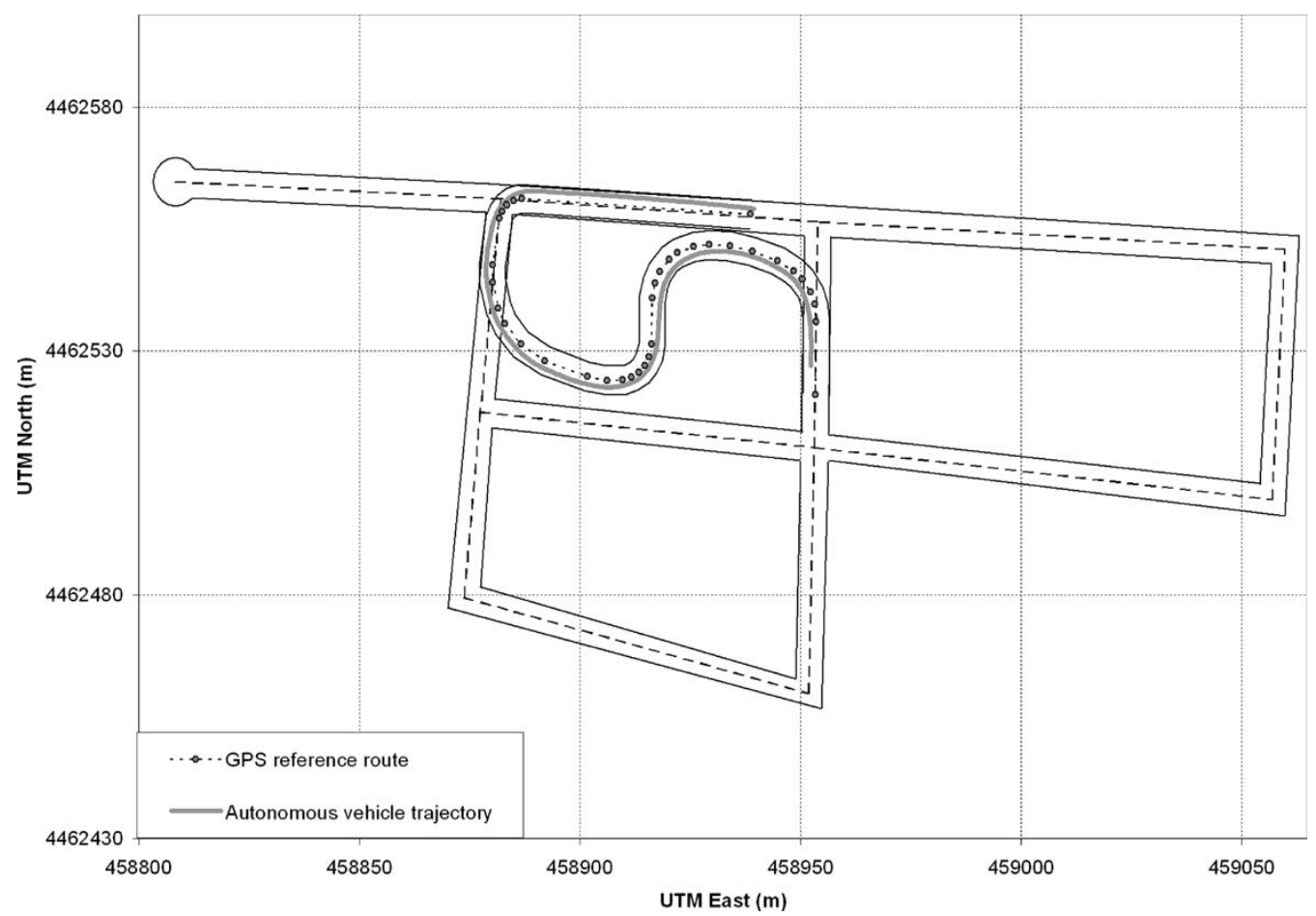

Fig. 8. Automatic-driving route experiment with diverse radius bends.
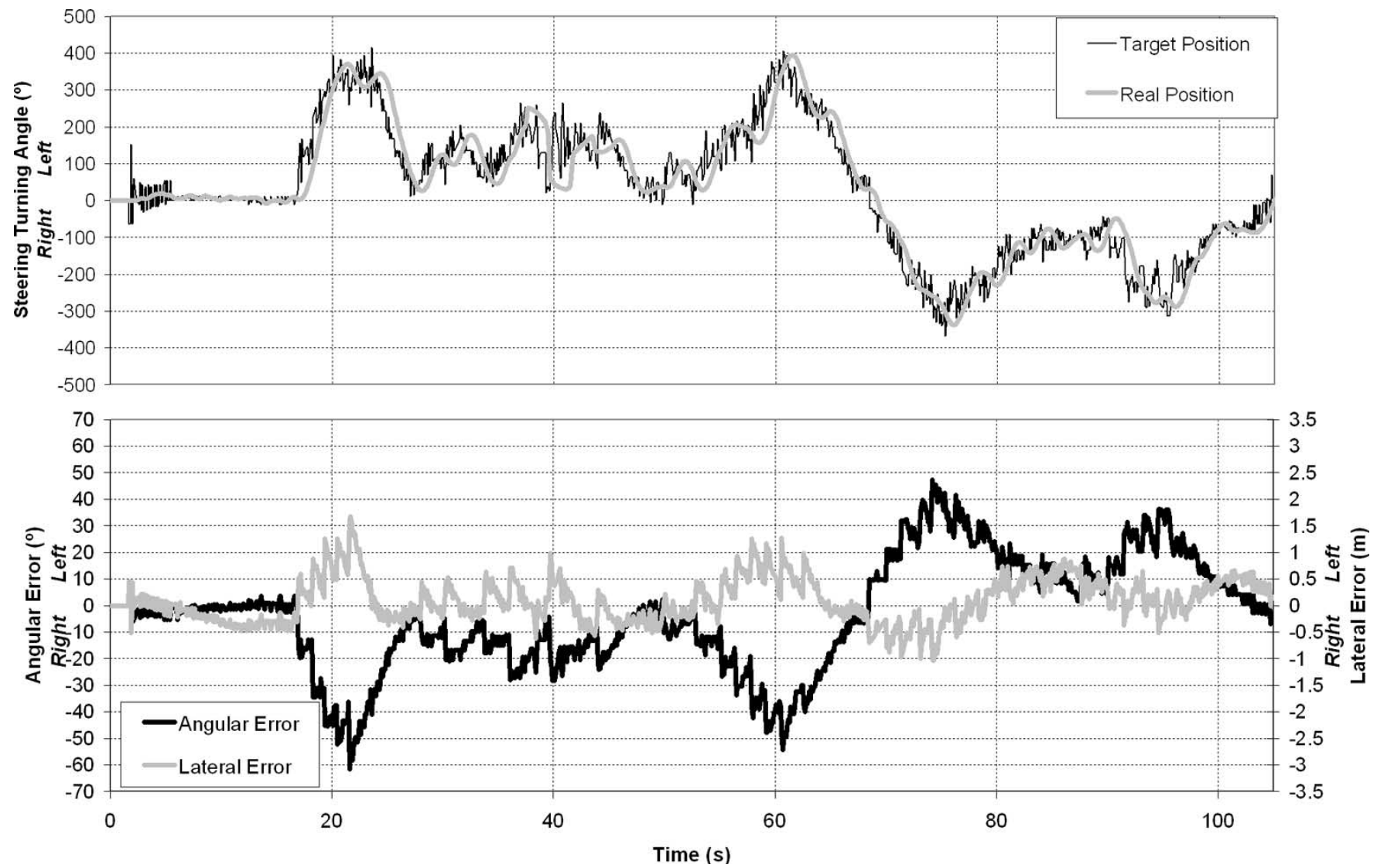

Fig. 9. Control response to the input variables during the second experimental track.

going out of lane, with the straight-controller average lateral and angular errors being $0.2 \mathrm{~m}$ and $0.85^{\circ}$, and the respective maxima $0.48 \mathrm{~m}$ and $3.58^{\circ}$.
Steering-control-system behavior is shown in Fig. 9. This is composed of two graphs: the input and output variables. The bottom graph depicts the values for the lateral and angular 
errors during the experiment. The route starts with straightroad tracking and a left turning on a short radius bend. In this situation, the angular error increases suddenly, and the steering-control action is a fast and wide wheel turning to keep to the trajectory. After a short straight-road segment, there are two broad left turnings, in which the angular error increases slowly as the control action does. After that, there is a right bend with a long radius. This causes the angular error and its respective control action to change slowly from right to left. The lateral error remains at near zero throughout the entire route, fulfilling its mission to keep the vehicle in the center of its reference lane.

From the top graph in Fig. 9, we can see that the system response to the turning angle stated by the fuzzy controller is optimum, and the steering tracking error is minimum, being enough to keep the vehicle in the reference route. This demonstrates the sound operation of the low-level PID controller. Videos showing the behavior of the presented system are available in [31].

\section{CONCLUSION}

In this paper, we have presented a control system for managing autonomous-vehicle steering. This control system is based on the cascade-control paradigm. In this case study, we exploit the synergy between a fuzzy controller for the outer control loop (high level) and a classical proportional-integral differential (PID) for the inner control loop (low level).

The fuzzy controller mimics human behavior, which rules out the need to design complex mathematical models or piecewise linear models that are unable to deal with nonlinear behavior. The fuzzy controller is designed and tuned according to a verbal description of driver experience. The PID controller provides functionality to a well-known regulating system so that a motor attached to the steering bar obeys the commands of the fuzzy controller.

The use of global-navigation satellite systems applied to automatic vehicle guidance has also proved to be a powerful technique that enables absolute positioning with a high nearreal-time accuracy.

Some automatic-driving experiments have been run with these systems, showing that the proposed cascade architecture behaves correctly and the related controllers are accurate.

These findings suggest that the research into autonomous vehicles within the intelligent transportation systems (ITSs) field is a short-term reality and a promising research area, and these results constitute the starting point for future developments. New sensory systems and sensory fusion is to be explored to plug additional information into the control system. Likewise, further studies will be conducted to refine and redesign the inner control system on the basis of fuzzy-logic- and neural-networkbased approaches. Furthermore, other maneuvers will be considered in the rule base of the outer control system to make the driving system capable of dealing with all driving situations.

\section{ACKNOWLEDGMENT}

The authors would like to thank Ministerio de Fomento and Ministerio de Educación. A especially thank to Citroën España
S.A., for without its collaboration, this work would not have been achieved.

\section{REFERENCES}

[1] European Transport Policy for 2010: Time to Decide, White Paper, Luxembourg: Office for Official Publications of the Eur. Communities, 2001. L-2985.

[2] J. E. Naranjo, C. González, J. Reviejo, R. García, and T. de Pedro, "Adaptive fuzzy control for inter-vehicle gap keeping," Special Issue on Adaptive Cruise Control, IEEE Trans. Intell. Transp. Syst., vol. 4, no. 3, pp. 132-142, Sep. 2003.

[3] - "A throttle \& brake fuzzy controller: Towards the automatic car," in Lecture Notes on Computer Science, vol. 2809. Berlin, Germany: Springer-Verlag, Jul. 2003, pp. 291-301.

[4] E. Dickmanns, "The development of machine vision for road vehicles in the last decade," in Proc. IEEE Intelligent Vehicles Symp., Versailles, France, 2002, pp. 268-281.

[5] J. Ackermann, T. Bünte, and D. Odenthal, "Advantages of active steering for vehicle dynamics control," in Proc. 32nd Int. Symp. Automotive Technology and Automation, Vienna, Austria, 1999, pp. 263-270.

[6] T. Endo, K. Iwazaki, and Y. Tanaka, "Development of reverse parking assist with automatic steering," in Proc. ITS Congr., Madrid, Spain, Nov. 2003.

[7] W. Ijiri and T. Tsutsui, "Technical trends of electric power steering systems," Koyo Eng. J. English Edition, no. 162E, pp. 27-31, 2003.

[8] S. Tsugawa, T. Yatabe, T. Hirose, and S. Matsumoto, "An automobile with artificial intelligence," in Proc. 6th Int. Joint Conf. Artificial Intelligence (IJCAI), Tokyo, Japan, 1979, pp. 893-895.

[9] S. Kato, S. Tsugawa, K. Tokuda, T. Matsui, and H. Fujiri, "Vehicle control algorithms for cooperative driving with automated vehicles and intervehicle communications," IEEE Trans. Intell. Transp. Syst., vol. 3, no. 3, pp. 155-161, Sep. 2002.

[10] A. Broggi, M. Bertozzi, A. Fascioli, and G. Conte, Automatic Vehicle Guidance: The Experience of the ARGO Autonomous Vehicle. Singapore: World Scientific, 1999.

[11] A. Broggi, M. Bertozzi, A. Fascioli, C. G. Lo Bianco, and A. Piazzi, "The ARGO autonomous vehicle's vision and control systems," Int. J. Intell. Control Syst., vol. 3, no. 4, pp. 409-441, Dec. 1999.

[12] S. Chaib, M. S. Netto, and S. Mammar, "H:, Adaptive, PID and fuzzy control: A comparison of controllers for vehicle lane keeping," in Proc. IEEE Intelligent Vehicles Symp., Parma, Italy, Jun. 2004, pp. 139-144.

[13] D. Pomerleau, "RALPH: Rapidly adapting lateral position handler," in Proc. IEEE Intelligent Vehicles Symp., Detroit, MI, 1995, pp. 506-511.

[14] R. Rajamani, H.-S. Tan, B. K. Law, and W.-B. Zhang, "Demonstration of integrated longitudinal and lateral control for the operation of automated vehicles in platoons," IEEE Trans. Contr. Syst. Technol., vol. 8, no. 4, pp. 695-708, Jul. 2000.

[15] J. Farrell, H.-S. Tan, and Y. Yang, "Carrier phase GPS-aided INS based vehicle lateral control," ASME J. Dyn. Syst. Meas. Control, vol. 125, No. 3, pp. 339-353, Sep. 2003.

[16] H.-S. Tan, B. Bougler, J. A. Farrell, and Y. Yang, "Automatic vehicle steering controls: DGPS/INS and magnetic markers," in Proc. Amer. Control Conf., Denver, CO, Jun. 2003, pp. 60-65.

[17] H.-S. Tan, B. Bougler, and W.-B. Zhang, "Automatic steering based on roadway markers-From highway driving to precision docking," Veh. Syst. Dyn., vol. 37, no. 5, pp. 315-339, Mar. 2002.

[18] T. Hessburg and M. Tomizuka, "Fuzzy logic control for lateral vehicle guidance," IEEE Control Syst., vol. 14, no. 4, pp. 55-63, Aug. 1994.

[19] J. M. Mendel, "Fuzzy logic systems for engineering: A tutorial," Proc. IEEE, vol. 83, no. 3, pp. 345-377, Mar. 1995.

[20] M. Sugeno and M. Nishida, "Fuzzy control of a model car," Fuzzy Sets Syst., vol. 16, no. 2, pp. 103-113, Aug. 1985.

[21] M. A. Sotelo, F.-J. Rodriguez, and L. Magdalena, "VIRTUOUS: Visionbased road transportation for unmanned operation on urban-like scenarios," IEEE Trans. Intell. Transp. Syst., vol. 5, no. 2, pp. 69-83, Jun. 2004.

[22] U. Franke, D. Gavrila, S. Görzig, F. Lindner, F. Paetzold, and C. Wöhler, "Autonomous driving approaches downtown," IEEE Intell. Syst., vol. 13, no. 6, pp. 40-48, Nov. 1999.

[23] E. D. Dickmanns and A. Zapp, "A curvature-based schema for improving road vehicle guidance by computer vision," in Proc. SPIE Conf. Mobile Robots, Cambridge, MA, 1986, vol. 727, pp. 161-168.

[24] J. E. Naranjo, C. González, R. García, T. de Pedro, J. Revuelto, and J. Reviejo, "Fuzzy logic based lateral control for GPS map tracking," in Proc. IEEE Intelligent Vehicles Symp., Parma, Italy, Jun. 2004, pp. $397-$ 400. 
[25] S. Hong, M. H. Lee, S. H. Kwon, and H. H. Chun, "A car test for the estimation of GPS/INS alignment errors," IEEE Trans. Intell. Transp. Syst., vol. 5, no. 3, pp. 208-218, Sep. 2004.

[26] S. Edelkamp, S. Jabbar, and T. Willhalm, "Geometric travel planning," IEEE Trans. Intell. Transp. Syst., vol. 6, no. 1, pp. 5-16, Mar. 2005.

[27] LM628 Programming Guide, LM628/629 User Guide, National Semiconductor Corp., Santa Clara, CA, 1999.

[28] K. Åström and T. Hägglund, PID Controllers: Theory, Design and Tuning. Research Triangle Park, NC: Instrument Soc. America, 1995.

[29] J. E. Naranjo, C. González, R. García, T. de Pedro, and J. Revuelto, "Fine tuning for autonomous vehicle steering fuzzy control," in Applied Computational Intelligence. Blakenberge, Belgium: World Scientific, 2004, pp. $450-455$.

[30] M. C. Andrade, R. Garcia, and T. de Pedro, "Software tools to obtain a distributed universal fuzzy controller," in Proc. IEEE Int. Symp. Industrial Electronics (ISIE), Santiago, Chile, 1994, pp. 408-423.

[31] Autopia Program web page. [Online]. Available: http://www.iai.csic.es/ autopia

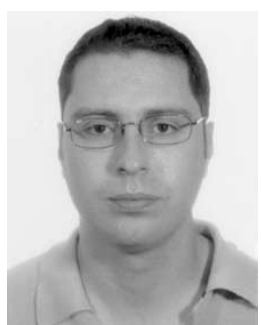

José E. Naranjo was born in Orense, Spain, in 1975. He received the B.E. and M.E. degrees in computer science engineering and the Ph.D. degree in computer science from the Polytechnic University of Madrid (UPM), Madrid, Spain, in 1998, 2001, and 2005, respectively.

Since 1999, he has been with the Industrial Computer Science Department, Instituto de Automática Industrial (CSIC), Madrid, Spain. His research interests include fuzzy-logic control and intelligent transportation systems (ITSs).

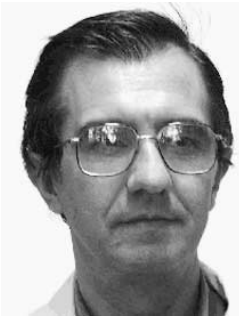

Carlos González (M'86) was born in Torrelavega (Cantabria), Spain, in 1947. He received the B.S. degree in physics from Madrid University, Madrid, Spain, in 1969, the M.S. degree in computer science from the University of California, Los Angeles (UCLA), in 1974, and the Ph.D. degree in physics from Madrid University in 1978.

He has been a Member of the staff of the Instituto de Automática Industrial, Madrid, since 1971, where he has always worked as a Software Specialist in automation projects.

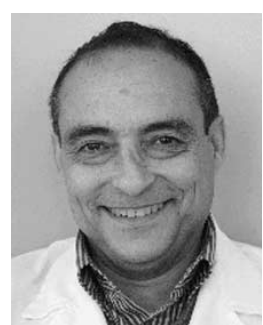

Ricardo García received the Ph.D. degree in physics from Bilbao University, Spain, in 1974

He was a Founder of Instituto de Automatica Industrial, Spanish Research Council, Madrid, Spain, where he has worked in intelligent robotics until now.

Dr. García was awarded in 2002 for the "Barreiros" research on automotive field prize for his Autopia project on intelligent transportation systems (ITSs).

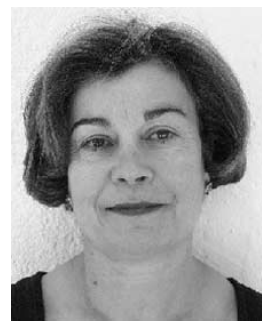

Teresa de Pedro received the Ph.D. degree in physics from the Universidad Complutense of Madrid, Madrid, Spain, in 1976.

Since 1971, she has been working on artificial intelligence applied to automation in the Instituto de Automática Industrial, Spanish Research Council, Madrid. Her current interest is on fuzzy models for unmanned vehicles. She is the Head of a Spanish team involved in the Integration of Sensors to Active Aided Conduction (ISAAC) project.

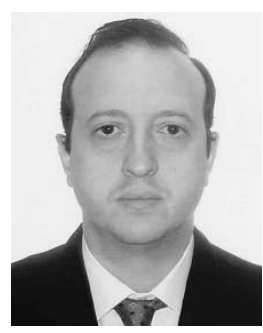

Rodolfo E. Haber was born in Santiago de Cuba, Cuba, in 1969. He received the B.E. degree with first-class honors in automatic control engineering from the Universidad de Oriente (UO), Santiago de Cuba, Cuba, in 1992. In 1995, he was the recipient of $\mathrm{Ph}$.D. grant supported by the Spanish Office for Scientific Cooperation (AECI) in Spain. He received the Excellent Cum Laude Ph.D. degree in industrial engineering from Universidad Politécnica de Madrid (UPM), Madrid, Spain, in 1999.

He joined the Spanish Council for Scientific Research (CSIC) in 1999, working in several research and development projects. In 1999, he was an Adjunct Professor of the Computer Engineering Department at the Escuela Politécnica Superior in the Universidad Autonoma de Madrid (UAM). His research interests include complex electromechanical processes, sensing systems, control theory and applications, hardware-software solutions, soft-computing techniques, classical and adaptive control, and supervisory control. 